\title{
Numeric Solution of the Fokker-Planck-Kolmogorov Equation
}

\author{
Claudio Floris \\ Department of Civil and Environmental Engineering, Politecnico di Milano, Milano, Italy \\ Email: claudio.floris@polimi.it
}

Received March 12, 2013; revised April 12, 2013; accepted April 19, 2013

Copyright (C) 2013 Claudio Floris et al. This is an open access article distributed under the Creative Commons Attribution License, which permits unrestricted use, distribution, and reproduction in any medium, provided the original work is properly cited.

\begin{abstract}
The solution of an $n$-dimensional stochastic differential equation driven by Gaussian white noises is a Markov vector. In this way, the transition joint probability density function (JPDF) of this vector is given by a deterministic parabolic partial differential equation, the so-called Fokker-Planck-Kolmogorov (FPK) equation. There exist few exact solutions of this equation so that the analyst must resort to approximate or numerical procedures. The finite element method (FE) is among the latter, and is reviewed in this paper. Suitable computer codes are written for the two fundamental versions of the FE method, the Bubnov-Galerkin and the Petrov-Galerkin method. In order to reduce the computational effort, which is to reduce the number of nodal points, the following refinements to the method are proposed: 1) exponential (Gaussian) weighting functions different from the shape functions are tested; 2) quadratic and cubic splines are used to interpolate the nodal values that are known in a limited number of points. In the applications, the transient state is studied for first order systems only, while for second order systems, the steady-state JPDF is determined, and it is compared with exact solutions or with simulative solutions: a very good agreement is found.
\end{abstract}

Keywords: Stochastic Differential Equations; Markov Vectors; Fokker-Planck-Kolmogorov Equation; Finite Element Numeric Solution; Modified Hermite Weighting Functions; Spline Interpolation

\section{Introduction}

It is widely recognized that many types of agencies acting on engineering structures and equipments have random characteristics. This is the case of earthquakes, wind load, wave load, electric current in a circuit, and so on. Thus, these agencies are to be described by means of the theory of stochastic processes. Moreover, in some cases, the structural properties themselves are uncertain, and must be considered as stochastic processes, which gives raise to a multiplicative or parametric excitation. In this way, the differential equations that govern the response become stochastic differential equations.

If the differential equations that govern the response are nonlinear or the excitation is parametric or both, the random response analysis is not an easy task, and mathematically exact solutions for the statistical characterization are not available in many cases. However, if the excitations are Gaussian white noise (WN) random processes, the system response is a vector Markov process, too: see [1-3].

If the initial state of a Markov process is known, then the joint probability density function (JPDF) of the states characterizes completely the stochastic process. The JPDF is the solution of a parabolic partial differential equation (PDE), the so-called Fokker-Planck-Kolmogorov (FPK) equation. This equation depends on time and on the actual values of the system states. However, if the system is damped and stable, a stationary state exists, which is characterized by the stationary JPDF $p(x)$, which does not depend on time. The FPK equation loses the dependence on time, and is called reduced FPK equation. A wide treatment of it can be found in $[3,4]$.

The severe limitations of the FPK equation approach resides in that exact analytical solutions are available in a restricted number of cases, and mostly in the steady state. In the transient state, analytical solutions were obtained for scalar systems only: the reader is referred to [4]. Along the years, much theoretical work has been done in order to solve the reduced FPK equation: [5-27]. Notwithstanding the considerable progress in this matter, two serious flaws remain: 1) in the case of multiplicative excitations, for the FPK equation to be solvable, restrictive relationships among the system parameters and the 
spectral densities of the excitations must be satisfied, and such requirements are rarely encountered in practical cases; 2) in several cases, no analytical solutions have been found, among the case of oscillators with a generic nonlinear damping mechanism.

For these reasons, the sixties numerical schemes of solution have been developed parallelly to the theoretical studies. These methods are: weighted residual method, eigenfunction expansion, finite differences, variational principles, and finite element (FE) method.

In the weighted residual method [28-33], the functional form of the JPDF is chosen a priori: a mixture of Gaussian functions or the exponential of a polynomial in the state variables is the most usual choice in both unknown coefficients' appearance. The approximate form of the JPDF cannot satisfy the FPK equation exactly so that a residual error arises. It is imposed that the projection of this into a set of weighting functions vanishes. In this way, a system of equations in the unknown coefficients is obtained, and it must be solved. Muscolino, Ricciardi and Vasta's method [29] is notable as it gives raise to an expression of the JPDF in which the coefficients are linear functions of the response statistical moments.

In the eigenfunction expansion method [34-38], the non-stationary JPDF of the system states is expressed as a truncated series of orthogonal functions, which are functions that form a complete orthonormal basis in a Hilbert space [39]. If the eigenfunctions of the FPK equation were available in all cases, this representation would be exact, but in general they are not known, and their computation even in an approximate numerical form is often cumbersome. Thus, the different authors adopt different strategies: in [35,38], the orthogonal functions are chosen a priori, and in order to determine the coefficients of the series, the weighted residual method is used. In [34,36,37], a perturbative approach is used.

Roberts use the finite difference method [40] to solve the FPK equation for the one-dimensional PDF of the energy envelope. In other words, there is only a spatial variable beyond the time variable, which is a limitation.

In [41], two types of variational approaches are presented, both of which are aimed at finding approximate values of the non-zero eigenvalues of the FPK operator (the first eigenvalue is zero, and it corresponds to the steady state PDF). The former one constructs an approximate Hermitian operator, and the other is based on a perturbation expansion. The applications are confined to the steady state. In [42], the variational approach gives raise to an iterative procedure. The applications regard the transient state of scalar systems.

The approximate solution of PDE's by means of the finite element method (FE) is a classic topics in numeric mathematics (e.g. see [43]). Probably, Bergman and
Heinrich [44-46] were the first who applied this method in the field of stochastic dynamics. In the above cited references, they did not analyze the FPK equation but the Pontriagin-Vitt equation in the moments of the first passage time of a level $\bar{x}$ from the displacement $X$ of a second order oscillator (as regards the Pontriagin-Vitt equation see [3], Chap. 8); however, the principles are the same. Then, the FE method was applied to the FPK equation: [47-54]. Differently from the weighted residual method in which the transition JPDF of the state vector has the same approximate form in the whole state space, in the FE method, the state space is discretized into elements inside which the JPDF varies according to the shape or trial functions that have been selected previously, and depending on the nodal values (see later). In [47-54], linear shape functions are used, which insure $C^{0}$ continuity only. Vice versa, as the FPK equation [Equations (6) and (7)] contains second derivatives, it would require $C^{1}$ continuity. The problem is overcomed by combining the FE method with the weighted residual method: the residual error is made orthogonal to a weight function in such a way that the coefficients of a linear system in the nodal values are computed. Fundamentally, there are two versions of the FE method for solving the FPK equation: the Bubnov-Galerkin and the PetrovGalerkin method. In the former, the weighting functions are equal to the trial functions. Vice versa, in the latter, weighting and trial functions are different, in general the weighting functions being non linear. This version is believed more suitable for convective problems [44,45,49, 52]. However, in the steady state, that is when solving the reduced FPK equation, there is no diffusion of probability. With the exception of $[49,52,53]$ in the above mentioned references, the applications regard the steady state because of the charge for computation. Some improvements of the method were proposed: in [51], an adaptive grid generation is used; in [53], a multi-scale FE method is fitted to the present problem; in [54], the solution is improved by means of a local hp-refinement of the mesh.

The present paper is organized as follows: Sec. 2 is devoted to the FPK equation. Sec. 3 treats the FE method for solving the FPK equation: first, its theoretical derivation is reviewed, and some topics are discussed. In detail, it is shown that: 1) to interpolate the nodal, values with splines of degree higher than those of the shape functions may improve the solution substantially; 2) in some cases the use of weighting functions different from the shape functions yields a notable computing time saving. The last two sections are devoted to the applications and conclusions respectively. In order to contain the computing time, the transient state is studied for first order systems only, while for second order systems the steady-state JPDF is determined. 


\section{The Fokker-Planck-Kolmogorov Equation}

Let $g(X(t))$ an arbitrary function of a stochastic process $X(t)$, which at its turn is solution to the stochastic differential equation

$$
\mathrm{d} X(t)=a(X(t), t)+b(X(t), t) \mathrm{d} B\left(X\left(t_{0}\right)=X_{0}\right),
$$

where $B(t)$ is a unit Wiener process, $a(X(t), t)$ is the drift term, and $b(X(t), t)$ is the diffusion term. As the latter depends on $X(t)$, that is the excitation is multiplicative, it is intended that $a(X(t), t)$ includes the so-called Wong-Zakai-Stratonovich corrective term $[55,56]$, which reads as

$$
a(X(t), t)=a^{*}(X(t), t)+\frac{1}{2} \frac{\partial b(X(t), T)}{\partial X} b(X(t), t),
$$

where $a^{*}(X(t), t)$ is the originary drift term before correction.

Now, we apply Itô's differential rule [57-59] to the first derivative of the stochastic expectation of $g$ :

$$
\frac{\mathrm{d}}{\mathrm{d} t} E\{g\}=E\left\{a \frac{\partial g}{\partial x}+\frac{b^{2}}{2} \frac{\partial^{2} g}{\partial x^{2}}\right\} .
$$

Expressing the expectations with respect the conditional PDF $p\left(x, t \mid x_{0}, t_{0}\right)$ of $X$, we obtain

$$
\begin{aligned}
& \frac{\mathrm{d}}{\mathrm{d} t} \int_{-\infty}^{\infty} g p\left(x, t \mid x_{0}, t_{0}\right) \mathrm{d} x \\
& =\int_{-\infty}^{\infty}\left[a \frac{\partial g}{\partial x}+\frac{b^{2}}{2} \frac{\partial^{2} g}{\partial x^{2}}\right] p\left(x, t \mid x_{0}, t_{0}\right) \mathrm{d} x
\end{aligned}
$$

Integrating Equation (4) by parts, and taking into account that the PDF $p$ and its first derivative tend to zero as $x$ tends to infinity; we obtain

$$
\begin{aligned}
& \int_{-\infty}^{\infty} g(x) \frac{\partial p}{\partial t} \mathrm{~d} x \\
& =\int_{-\infty}^{\infty}\left\{-\frac{\partial}{\partial x}[a(x, t) p]+\frac{1}{2} \frac{\partial^{2}}{\partial x^{2}}\left[b^{2}(x, t) p\right]\right\} g(x) \mathrm{d} x
\end{aligned}
$$

As $g(x)$ is quite arbitrary, it may be assumed $g(x)=1$, which yields:

$$
\frac{\partial f}{\partial t}=-\frac{\partial}{\partial x}[a(x, t) p]+\frac{1}{2} \frac{\partial^{2}}{\partial x^{2}}\left[b^{2}(x, t) p\right] .
$$

Equation (6) is the Fokker-Planck-Kolmogorov equation (or forward Kolmogorov equation) in the unknown transition JPDF of the scalar process $X(t)$. It is a parabolic PDE, which must satisfy suitable initial and boundary conditions. If the initial state is deterministic, then $p\left(x, t_{0} \mid x_{0}, t_{0}\right)=\delta\left(x-x_{0}\right)$, where $\delta(\cdot)$ is Dirac delta. At infinite boundaries $p\left(x, t_{0} \mid x_{0}, t_{0}\right)$ must be zero.

Now, Equation (1) be replaced by the analogous vector equation $\mathrm{d} \boldsymbol{X}(t)=\boldsymbol{a}(X(t), t)+\boldsymbol{G}(X(t), t) \mathrm{d} \boldsymbol{B}$, where $\boldsymbol{X}$, and $\boldsymbol{a}$ are $n \times 1$ vectors, $\boldsymbol{G}$ is an $n \times m$ matrix, and $\boldsymbol{B}$ is an $m \times 1$ vector of Wiener processes with covariance matrix $\Gamma$. It can be demostrated that the FPK equation for the JPDF $p\left(\boldsymbol{x}, t \mid \boldsymbol{x}_{0}, t_{0}\right)$ of $\boldsymbol{X}(t)$, given $\boldsymbol{X}\left(t_{0}\right)=\boldsymbol{x}_{0}$, is

$$
\frac{\partial p}{\partial t}=-\frac{\partial}{\partial x_{i}}\left[a_{i}(x, t) p\right]+\frac{1}{2} \frac{\partial^{2}}{\partial x_{i} \partial x_{j}}\left[b_{i j}(x, t) p\right] .
$$

In Equation (7) the summation rule with respect to repeated indexes is implicitly adopted, and $b_{i j}=\left[\boldsymbol{G}^{t} \Gamma \boldsymbol{G}\right]_{i j}$. Equation (7) has an important physical interpretation. Define

$$
C_{j}=a_{j} p-\frac{1}{2} \cdot \frac{\partial\left(b_{j k} p\right)}{\partial x_{k}} .
$$

Then, Equation (7) is rewritten as

$$
\frac{\partial p}{\partial t}+\frac{\partial\left(C_{j}\right)}{\partial x_{j}}=0
$$

Equation (9) has the same form as the continuity equation of fluid mechanics. In this way, $C_{j}$ is the $j$-th component of the vector of the probability current, so that Equation (9) expresses the conservation of the probability. From that it is deducted that the $C_{j}$ 's must be zero at the boundaries of the existence domain, which in most cases is infinite; hence, at the boundaries the JPDF $p$ is zero. So, it is demonstrated that the FPK equation describes a convection problem.

Now, for the clarity's sake some solutions of the reduced (steady-state) FPK equation are given. The motion equation of a second order oscillator with nonlinear restoring force is

$$
\ddot{X}(t)+\beta \dot{X}(t)+F(X)=\sqrt{2 \pi K} W(t),
$$

where $W(t)$ is a unit strength Gaussian white noise. It is assumed that $F(x)$ is a conservative force, which is it is the derivative of a potential function $U(x)$ as

$$
F(x)=\frac{\mathrm{d} U(x)}{\mathrm{d} x} .
$$

Equation (10) is equivalent to the following two first order Itô's stochastic differential equations in the phase space:

$$
\left\{\begin{array}{l}
\mathrm{d} x_{1}=x_{2} \mathrm{~d} t \\
\mathrm{~d} x_{2}=\left[-\beta x_{2}(t)-F\left(x_{1}\right)\right] \mathrm{d} t+\sqrt{2 \pi K} \mathrm{~d} B(t)
\end{array},\right.
$$

where $x_{1}=X$ and $x_{2}=\dot{X}$. The FPK equation governing the stationary JPDF $p_{x_{1} x_{2}}$ is

$$
\pi K \frac{\partial^{2} p}{\partial x_{2}^{2}}-\frac{\partial\left(p x_{2}\right)}{\partial x_{1}}+\frac{\partial}{\partial x_{2}}\left[\left(\beta x_{2}+F\left(x_{1}\right)\right) p\right]=0,
$$

where $p=p_{x_{1} x_{2}}$, and the current values of the variables have been denoted with lower case letters. The solution 
to Equation (13) is [5]

$$
p_{X_{1} X_{2}}\left(x_{1}, x_{2}\right)=p_{X \dot{X}}(x, \dot{x})=C \cdot \mathrm{e}^{\left\{-\frac{\beta}{\pi K}\left[U(x)+\frac{\dot{x}^{2}}{2}\right]\right\}},
$$

where $C$ is a normalization constant.

In [5], the FPK equation is solved too for an $N$-degreeof-freedom ( $2 N$ states) system, whose motion equation have the form

$$
\begin{aligned}
& \ddot{X}_{i}(t)+\beta_{i} \dot{X}_{i}(t)+\frac{1}{M_{i}} \frac{\partial U}{\partial X_{i}} \\
& =\sqrt{2 \pi K_{i}} W_{i}(t) \quad(i=1,2, \cdots, N)
\end{aligned}
$$

where no summation has to be performed with respect to the index $i$; the white noises $W_{i}(t)$ are uncorrelated. The JPDF of the variables $x_{i}, \dot{x}_{i}$ is [5]

$$
\begin{aligned}
& p\left(x_{1}, \cdots, x_{N}, \dot{x}_{1}, \cdots, \dot{x}_{N}\right) \\
= & C \cdot \exp \left[-U\left(x_{1}, \cdots, x_{N}\right) \cdot\left(\sum_{i=1}^{N} \frac{\beta_{i}}{\pi K_{i} M_{i}}\right)-\frac{\beta_{i}}{\pi K_{i}} \frac{\dot{x}_{i}^{2}}{2}\right] .
\end{aligned}
$$

In Equation (5) the equipartition of the energy among the degree of freedoms is notable.

Liu [8] considers a class of $N$-degree-of-freedom nonlinear discrete dynamic systems, whose equations of motions are

$$
\begin{aligned}
& \ddot{X}_{i}+c_{i} \dot{X}_{i}\left[1+\varepsilon_{i} F_{i}\left(x_{1}, x_{2}, \cdots, x_{n} ; \dot{x}_{1}, \dot{x}_{2}, \cdots, \dot{x}_{n}\right)\right] \\
& +k_{i} X_{i}\left[1+\mu_{i} R_{i}\left(x_{1}, x_{2}, \cdots, x_{n} ; \dot{x}_{1}, \dot{x}_{2}, \cdots, \dot{x}_{n}\right)\right]=W_{i}(t)
\end{aligned}
$$

where $i=1,2, \cdots, N, c_{i}, \varepsilon_{i}, k_{i}, \mu_{i}$ are constant parameters, and the Gaussian white noise processes $W_{i}$ are characterized by $E\left[W_{i}\left(t_{1}\right) W_{j}\left(t_{2}\right)\right]=S_{i j} \delta_{i j}\left(t_{1}-t_{2}\right) \quad$ with $\delta_{i j}=$ Kronecker's delta. The coefficients of Equation (8) are:

$$
\begin{aligned}
& a_{i}=\dot{x}_{i} \\
& a_{i+1}=-c_{i} \dot{X}_{i}\left[1+\varepsilon_{i} F_{i}\left(x_{1}, x_{2}, \cdots, x_{n} ; \dot{x}_{1}, \dot{x}_{2}, \cdots, \dot{x}_{n}\right)\right] \\
& -k_{i} X_{i}\left[1+\mu_{i} R_{i}\left(x_{1}, x_{2}, \cdots, x_{n} ; \dot{x}_{1}, \dot{x}_{2}, \cdots, \dot{x}_{n}\right)\right] \\
& b_{i i}=b_{i j}=0 \quad b_{i+1 . i+1}=S_{i i}
\end{aligned}
$$

He gives the following solutions.

I-Under the hypothesis:

- the $C_{j}^{\prime} \mathrm{s}_{i}$ are the same for all the degree of freedom;

- the matrix $\left[b_{i j}\right]$ is not singular so that the inverse matrix $\Delta$ exists; the following relationships hold

$$
\begin{aligned}
& \frac{\partial}{\partial y_{\alpha}}\left\{\sum_{i} \Delta_{\beta i}\left(\sum_{j} \frac{\partial b_{i j}}{\partial y_{i}}-2 a_{i}\right)\right\} \\
& =\frac{\partial}{\partial y_{\beta}}\left\{\sum_{i} \Delta_{\alpha i}\left(\sum_{j} \frac{\partial b_{i j}}{\partial y_{i}}-2 a_{i}\right)\right\}
\end{aligned}
$$

where $y_{\alpha}, y_{\beta}=x_{k}$ if $\alpha, \beta=1,2, \cdots, N$, and $y_{\alpha}, y_{\beta}=\dot{x}_{k}$ if $\alpha, \beta=N+1, N+2, \cdots, 2 N$; the probability flux is null around the existence domain. In this case. the steady state PDF is given by

$$
\begin{aligned}
& p\left(y_{1}, y_{2}, \cdots\right) \\
& =C \exp \left\{-\int_{0}^{y_{1}} \cdots \int_{0}^{y_{2 N}}\left(\Delta_{k i} \frac{\partial b_{i j}}{\partial y_{j}}-2 \Delta_{k i} a_{i}\right) \mathrm{d} y_{k}\right\},
\end{aligned}
$$

where the summation with respect to the indexes $j$ and $k$ is implicit. Equation (20) may or may not result in a closed form depending on whether the integral is analitically evaluable.

II-The addenda $\frac{\partial C_{j}}{\partial x_{j}}$ in Equation (9) are singularly zero according to the relationships

$$
L_{j}\left[g_{j}\left(y_{j}\right) p+h_{j}\left(y_{j}\right) \frac{\partial p}{\partial y_{j}}\right]=0(i=1,2, \cdots, 2 N),
$$

where $L_{j}$ are first order differential operators, while $g_{j}$ and $h_{j}$ are nonlinear functions. Then, the JPDF is

$$
p\left(y_{1}, y_{2}, \cdots, y_{N}\right)=C \prod_{1}^{2 N} \exp \left[-\int_{0}^{y_{i}} \frac{g_{i}\left(u_{i}\right)}{h_{i}\left(u_{i}\right)} \mathrm{d} u_{i}\right] \text {. }
$$

In [13] by applying the principle of detailed balance, the steady state JPDF's of some notable oscillators are found. The first of them is

$$
\ddot{X}(t)+h(\Lambda) \dot{X}(t)+F(X)=\sqrt{2 \pi K} W(t),
$$

where $h(\Lambda)$ is a generic function of the mechanical energy of the oscillator, that is

$$
\Lambda=\frac{1}{2} \dot{x}^{2}+\int_{0}^{x} F(u) \mathrm{d} u .
$$

The steady-state JPDF is

$$
p(x, \dot{x})=C \exp \left[-\frac{1}{\pi K} \int_{0}^{\Lambda} h(u) \mathrm{d} u\right] .
$$

Consider the oscillator with parametric excitation

$$
\ddot{X}(t)+\left[h(\Lambda)+W_{2}(t)\right] \dot{X}(t)+\omega_{0}^{2}\left[1+W_{1}(t)\right]=W_{3}(t),
$$

where $h(\Lambda)$ is a function of the mechanical energy $\Lambda$, and the processes $W_{1}, W_{2}, W_{3}$ are uncorrelated Gaussian white noises with power spectral densities $K_{1}, K_{2}$, and $K_{3}$, respectively. The steady-state JPDF is

$$
p(x, \dot{x})=C \exp [-\phi(x, \dot{x})],
$$

where $\phi(x, \dot{x})$ is given by the equation

$$
\frac{\partial \phi(x, \dot{x})}{\partial \dot{x}}=\frac{\left[h(\Lambda)+\pi K_{2}\right] \dot{x}}{\pi\left(\omega_{0}^{4} K_{1} x^{2}+K_{2} \dot{x}^{2}+K_{3}\right)} .
$$


A more general case of FPK equation for an oscillator with parametric excitation of white noises is solved in [15] by applying the concept of generalized stationary potential. It is

$$
\ddot{X}(t)+h(X(t), \dot{X}(t))=f_{i}(X(t), \dot{X}(t)) W_{i}(t),
$$

where the summation rule with respect to a repeated index is valid, $f_{i}(X(t), \dot{X}(t))$ are nonlinear functions, and the white noises $W_{i}$ are characterized by the correlation $E\left[W_{i}(t) W_{j}(t+\tau)\right]=2 \pi K_{i j} \delta(\tau)$. The steady-state PDF has the form of Equation (27) with

$$
\phi(x, \dot{x})=-\ln \frac{\partial \Lambda}{\partial y}+\phi_{0}(\Lambda),
$$

where $\phi$ is the generalized stationary potential, $y=1 / 2 \dot{x}^{2}$, and $\phi_{0}$ a function of the mechanic energy. Equation (30) is valid under the condition

$$
\begin{aligned}
& h(x, \dot{x})=\pi \dot{x} K_{i j} f_{i}(x, \dot{x}) f_{j}(x, \dot{x})\left[\Lambda_{y} \phi_{0}^{\prime}(\Lambda)-\frac{\Lambda_{y y}}{\Lambda_{y}}\right], \\
& -\pi k_{i j} f_{i}(x, \dot{x}) \frac{\partial f_{j}(x, \dot{x})}{\partial \dot{x}}+\frac{\Lambda_{x}}{\Lambda_{y}}+\frac{D(x)}{\Lambda_{y}} \mathrm{e}^{\phi_{0}(\Lambda)}
\end{aligned}
$$

where $\Lambda_{x}, \Lambda_{y}, \Lambda_{y y}$ are the derivatives of $\Lambda$ with respect to $x, y$, and to $y$ twice, respectively; $\phi_{0}^{\prime}$ is the derivative of $\phi$ with respect to $\lambda$, that is the generic value of $\Lambda$.

At this stage the functions $D(x)$ and $\phi_{0}(\Lambda)$ are still unknown: they can be identified by applying Equation (31); see the examples in [15]. It is notable that Equation (31) is a very restrictive relation between the system nonlinearity and the excitation parameters, which is rarely met in practice.

Zhu found the exact stationary solution of the FPK equation pertaining to several classes of nonlinear dynamic systems $[18,20,26,27]$. First, we recall the case of the following nonlinear stochastic system:

$$
\begin{aligned}
& \ddot{X}(t)+a \dot{X}\left[H_{y} f(H)-\frac{H_{y y}}{H_{y}}\right]+\frac{H_{x}}{H_{y}} . \\
& =b g_{i}(H) W_{i}(t)
\end{aligned}
$$

In Equation (32) $a$ and $b$ are constants, $y=\dot{x}^{2} / 2$, each subscript $x$ or $y$ means partial derivative,

$H=H(x, y)$ is the first integral of the oscillator $\ddot{x}+H_{x} / H_{y}=0$, while $f$ and $g_{i}$ are nonlinear functions of $H$. The steady state JPDF of $X$ and $\dot{X}$ has the general form $p(x, \dot{x})=\phi(H) \cdot H_{y}$. If the ratio $H_{y y} / H_{y}^{2}$ is constant or depends only on $H$, the function $\phi(H)$ can be determined giving raise to

$$
p(x, \dot{x})=C H_{y}\left(K_{i j} g_{i} g_{j}\right)^{-\frac{1}{2}} \cdot \exp \left[-\int_{0}^{H} F(u) \mathrm{d} u\right],
$$

where the $K_{i j}$ 's define the correlations among the white noises, that is $E\left[W_{i}(t) W_{j}(t+\tau)\right]=2 K_{i j} \delta(\tau)$, and

$$
F(u)=\frac{H_{y y}}{H_{y}^{2}}+\frac{a\left[f(u)-H_{y y} / H_{y}^{2}\right]}{b^{2} K_{i j} g_{i}(u) g_{j}(u)} .
$$

Another class of dynamic systems for which the associated FPK equation has been extensively studed is that of stochastically excited and dissipated Hamiltonian systems with $n$-degree-of-freedom $[17,19,20,26,27]$ :

$$
\begin{aligned}
& \dot{Q}_{i}=\frac{\partial \hat{H}}{\partial P_{i}} \\
& \dot{P}_{i}=-\frac{\partial \hat{H}}{\partial P_{i}}-c_{i j} \frac{\partial \hat{H}}{\partial P_{j}}+f_{i k} W_{k}(t),
\end{aligned}
$$

where $Q_{i}$ and $P_{i}$ are generalized displacements and momenta, respetively; $\hat{H}=\hat{H}(\boldsymbol{Q}, \boldsymbol{P})$ is a twice differentiable Hamiltonian; $c_{i j}=c_{i j}(\boldsymbol{Q}, \boldsymbol{P})$ are differentiable functions; $f_{i k}=f_{i k}(\boldsymbol{Q}, \boldsymbol{P})$ are twice differentiable functions; $i, j=1,2, \cdots, n ; k=1,2, \cdots, m$; and $W_{k}$ are Gaussian white noises with correlation functions

$$
E\left[W_{k}(t) W_{l}(t+\tau)\right]=2 D_{k l} \delta(\tau) .
$$

In the second of Equations (35) the excitations are multiplicative: hence, for transforming them into Itô type stochastic differential equations, they are to be modified by means of the Wong-Zakai-Stratonovich corrective terms $[55,56]$. The transformed equations in incremental form are

$$
\begin{aligned}
& \mathrm{d} Q_{i}=\frac{\partial H}{\partial P_{i}} \mathrm{~d} t \\
& \mathrm{~d} P_{i}=\left(-\frac{\partial H}{\partial Q_{i}}-m_{i j} \frac{\partial H}{\partial P_{j}}\right) \mathrm{d} t+f_{i k} \mathrm{~d} B_{k},
\end{aligned}
$$

where in general $H \neq \hat{H}$ and $m_{i j} \neq c_{i j}$.

The FPK equation associated with Equation (36) is

$$
\begin{aligned}
& -\frac{\partial}{\partial q_{i}}\left(\frac{\partial H}{\partial p_{i}} p\right)+\frac{\partial}{\partial p_{i}}\left(\frac{\partial H}{\partial q_{i}} p\right)+\frac{\partial}{\partial p_{i}}\left(m_{i j} \frac{\partial H}{\partial p_{j}} p\right), \\
& +\frac{1}{2} \frac{\partial^{2}}{\partial p_{i} \partial p_{j}}\left(b_{i j} p\right)=0
\end{aligned}
$$

where $b_{i j}=2\left[\boldsymbol{F D F}^{\mathrm{T}}\right]_{i j}, \boldsymbol{F}=\left[f_{i k}\right], \boldsymbol{D}=\left[D_{k l}\right]$. The steady-state PDF solution to Equation (37) has the form

$$
p(\boldsymbol{q}, \boldsymbol{p})=C \exp (-\phi),
$$

where the vectors $\boldsymbol{q}$ and $\boldsymbol{p}$ contain the generalized displacements and momenta, respectively. If the conservative Hamiltonian system $\dot{q}_{i}=\partial H / \partial p_{i}, \dot{p}_{i}=\partial H / \partial q_{i}$ is non-integrable, that is its first integral is the Hamiltonian itself [60], the function $\phi$ depends on $H$ only, and it is 
obtained by solving a system of first-order linear ordinary differential equations. If there are $n$ independent integrals of motion $H_{1}=H, H_{2}, \cdots, H_{n}, \phi$ depends on these, and it is the solution of $n$ first order PDE's. For both cases the reader is referred to [20] for the details.

\section{Finite Element Method Formulation}

\subsection{General Formulation}

In the finite element (FE) procedure the state space is discretized into a number of finite regions: the finite element mesh consists of a grid of points at which the JPDF $p(\mathbf{z}, t)$ is to be computed. It must be emphasized that in general the JPDF $p$ exists in an infinite hyperdomain. If the FE procedure is applied to an infinite domain, three different approaches are possible: 1) the inner region of the domain is divided into finite elements, while the outer region is discarded since the quantity to be computed is assumed negligible therein; 2) the inner region of the domain is divided into finite elements, while the outer region is modelled by infinite elements that extend to infinity; 3) the outer region is modelled using boundary elements or series solution. The first approach is used herein as the probability density functions decay to small or very small values, when the variables are larger than a few standard deviations. An estimate of the standard deviations of the response variables is obtained easily by applying the equivalent linearization method [61].

Inside an element $s$ (Figure 1) the JPDF $p(\mathbf{z}, t)$ is approximated by $p_{(e l-s)}$, which is given by

$$
p_{(e l-s)}(\mathbf{z}, t)=\sum_{1}^{N_{n o}}{ }_{k} p_{k}^{(s)}(t) \cdot N_{k}(\mathbf{z}) .
$$

In Equation (39) $N_{n o}$ are the nodes of the element, where the JPDF assumes the nodal values $p_{k}^{(s)}$. Clearly, inside an element the JPDF varies according to the shape functions $N_{k}(\mathbf{z})$. The nodal values of contiguous elements must be equal.

If linear shape functions are selected, the values of the JPDF at the nodes are the only as unknowns. On the other hand, linear shape functions yield $C^{0}$ continuity, while $C^{1}$ continuity is required as the FPK equation is of second order. This problem is overcome by considering a weak solution of it. Define the operators

$$
L_{1}=-\frac{\partial}{\partial \mathbf{z}_{i}}\left[a_{i}(\mathbf{z})\right] \quad L_{2}=\frac{1}{2} \frac{\partial^{2}}{\partial \mathbf{z}_{i} \partial \mathbf{z}_{j}}\left[b_{i j}(\mathbf{z})\right] .
$$

For any weighting function $\phi(\mathbf{z})$ it must be

$$
\int_{\Omega} \phi \frac{\partial p}{\partial t} \mathrm{~d} \mathbf{z}=\int_{\Omega} \phi L_{2}[p] \mathrm{d} \mathbf{z}+\int_{\Omega} \phi L_{1}[p] \mathrm{d} \mathbf{z},
$$

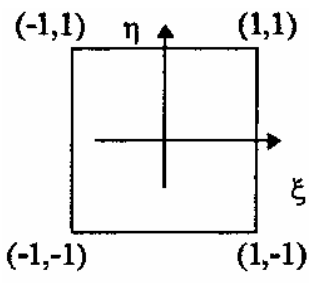

Figure 1. Four-node rectangular finite element.

in which the dependence on $\boldsymbol{z}$ has been omitted, and $\Omega$ denotes the domain of existence of $p(\mathbf{z}, t)$. Integrating the right-hand-side of Equation (41) by parts, and accounting for the fact that $p \rightarrow 0$ as $|\mathbf{z}| \rightarrow \infty$, we obtain the weak form of the FPK equation as

$$
\int_{\Omega} \phi \frac{\partial p}{\partial t} \mathrm{~d} \mathbf{z}=\int_{\Omega} a_{i} p \frac{\partial \phi}{\partial z_{i}} \mathrm{~d} \mathbf{z}-\frac{1}{2} \int_{\Omega} b_{i j} \frac{\partial \phi}{\partial z_{i}} \frac{\partial p}{\partial z_{j}} \mathrm{~d} \mathbf{z}
$$

Equation (42) is obtainable also with a variational formulation [48].

If Equation (39) is inserted in (42), and the integration over the domain $\Omega$ is replaced by the sum of the integrals over each element, we obtain the matrix system

$$
\boldsymbol{C} \dot{\boldsymbol{p}}(t)+\boldsymbol{K} \boldsymbol{p}(t)=\mathbf{0},
$$

in which $\boldsymbol{p}(t)$ is an $N_{n o} \times 1$ column vector containing the nodal values, and $\mathbf{0}$ is an $N_{n o} \times 1$ column vector whose components are zero.

The various entries of the $N_{n o} \times N_{\text {no }}$ matrices $\boldsymbol{C}, \boldsymbol{K}$ depend on the choice that has been made for the weighting functions $\phi(z)$. Bergman and Heinrich [45], Langtangen [48], Köylüoglu and collaborators [50] use weighting functions different from the shape functions, while the other authors use weighting functions equal to the shape functions. This choice, which is referred as BubnovGalerkin FE method, gives

$$
\begin{array}{r}
k_{\ell m}=\int_{E} a_{i} N_{\ell} \frac{\partial N_{m}}{\partial z_{i}} \mathrm{~d} \mathbf{z}-\frac{1}{2} \int_{E} \frac{\partial N_{\ell}}{\partial z_{i}} \frac{\partial\left(N_{m} b_{i j}\right)}{\partial z_{j}} \mathrm{~d} \mathbf{z}, \\
c_{\ell m}=\int_{E} N_{\ell} N_{m} \mathrm{~d} \mathbf{z} \quad\left(\ell, m=1, \cdots, N_{\text {no }}\right),
\end{array}
$$

where $k_{\ell m}$ is a generic element of the local "stiffness" matrix, $c_{\ell m}$ is an element of the local matrix multiplying $\dot{\boldsymbol{p}}^{(s)}$, and $E$ denotes that the integration is performed over the element. Transformation of each element matrix into global coordinates and summation over the elements allows the construction of the matrices $\boldsymbol{C}$ and $\boldsymbol{K}$.

If the shape and weighting functions are chosen to be different, Equations $(44,45)$ are replaced by, respectively

$$
\begin{gathered}
k_{\ell m}=\int_{E} a_{i} N_{\ell} \frac{\partial \phi_{m}}{\partial z_{i}} \mathrm{~d} \mathbf{z}-\frac{1}{2} \int_{E} \frac{\partial N_{\ell}}{\partial z_{i}} \frac{\partial\left(\phi_{m} b_{i j}\right)}{\partial z_{j}} \mathrm{~d} \mathbf{z}, \\
c_{\ell m}=\int_{E} \phi_{\ell} N_{m} \mathrm{~d} \mathbf{z} .
\end{gathered}
$$




\subsection{Refinements of the Method}

Computer codes have been written and implemented to solve both the transient and the reduced FPK equations (in this case Equation (43) reduces to $\boldsymbol{K p}=\mathbf{0}$ ): because of brevity's sake the features of these codes are not expounded. It is only recalled that Equation (43) is solved by adopting a forward finite difference scheme. When the shape and weighting functions are equal, in the case of a rectangular element (Figure 1) the functions in local coordinates $\xi, \eta$ are

$$
\begin{aligned}
& N_{1}(\xi, \eta)=\phi_{1}(\xi, \eta)=1 / 4(1-\xi)(1-\eta) \\
& \left.N_{2}(\xi, \eta)\right)=\phi_{2}(\xi, \eta)=1 / 4(1+\xi)(1-\eta) \\
& N_{3}(\xi, \eta)=\phi_{3}(\xi, \eta)=1 / 4(1+\xi)(1+\eta) \\
& N_{4}(\xi, \eta)=\phi_{4}(\xi, \eta)=1 / 4(1-\xi)(1+\eta)
\end{aligned}
$$

This choice has the undoubted advantage of making the solutions of the integrals faster (obviously, the integrals are numerically evaluated).

If $\phi_{i}$ is different from $N_{\mathrm{i}}$ are, the problem of choosing the weighting must be $\phi_{i}$ solved. No theoretical rules exist for doing it. Thus, the choice was based on empirical considerations, but keeping into account that each weighting function must be 1 in the corresponding node and zero on the sides not converging in it. The final $\phi_{i}$ 's are:

$$
\begin{aligned}
& \phi_{1}(\xi, \eta)=N_{1}(\xi, \eta) \cdot \exp \left[-a^{2}(1+\xi)^{2}-b^{2}(1+\eta)^{2}\right] \\
& \phi_{2}(\xi, \eta)=N_{2}(\xi, \eta) \cdot \exp \left[-a^{2}(1-\xi)^{2}-b^{2}(1+\eta)^{2}\right] \\
& \phi_{3}(\xi, \eta)=N_{3}(\xi, \eta) \cdot \exp \left[-a^{2}(1-\xi)^{2}-b^{2}(1-\eta)^{2}\right] \\
& \phi_{4}(\xi, \eta)=N_{4}(\xi, \eta) \cdot \exp \left[-a^{2}(1+\xi)^{2}-b^{2}(1-\eta)^{2}\right]
\end{aligned}
$$

in which the constants $a, b$ depend on the transformation from global to local coordinates, and are assumed differently in the different cases. Thus, the $\phi_{i}$ 's are multiplied by Gaussian like functions that cause them to decay fast from the nodes.

Computing the JPDF of the state variables is not the final stage of the analysis of a random dynamic system. In fact, one needs to obtain the response statistics such as marginal densities, statistical moments, and mean upcrossing rate (MUR) functions. The former can be directly obtained as geometrical sections of the hypersurface having $p(\mathbf{z}, t)$ as equation. However, the other quantities require integration: of the marginal PDF as regards the response moments, and of the JPDF of $X, \dot{X}$ as regards the MUR function.

In many cases, accepting or rejecting a JPDF or a PDF obtained by means of the FE method is decided by a visual inspection. On the contrary, a sound quantitative criterion of acceptance must be based on the errors that the computed statistics have with respect to the exact or simulative ones. In this paper, an improvement of the method is proposed in order to compute better estimates of the statistics without increasing the number of nodes. The nodal values are interpolated by both quadratic and cubic splines, the former choice bringing to the well known Cavalieri-Simpson's rule of integration.

\section{Applications}

The present section is devoted to the presentation of the results that are obtained applying the FE method to the solution of the FPK equations of different stochastic differential equations. In order to limit the computing time, the transient state is analyzed only for two scalar systems. In fact, the solution of the complete Equation (43) is much more time consuming as it requires a multi-fold discretization in the state coordinate and in time. On the contrary, the solution of the steady-state equation $\mathbf{K p}=\mathbf{0}$ can be easily performed even on a normal personal computer. Two-state systems are analyzed in this case. The results of the Bubnov-Galerkin version of the FE method are compared with those of the Petrov-Galerkin one, which is indicated as WRM. In computing the staistical moments, the effets of the spline interpolation of the nodal values are highlited.

\subsection{Scalar Systems}

The feasibility of the FE computer code that has been implemented is firstly tested by determining the time evolution of the PDF of the solution $X$ of two scalar systems excited by a stationary Gaussian white noise. It has been chosen the Langevin equation with linear or nonlinear drift term. In the first case the PDF of $X$ is Gaussian. The equation of motion are

$$
\begin{gathered}
\dot{X}=-a X+\sqrt{2 \pi K} W(t) \\
\dot{X}=-a X-b X^{3}+\sqrt{2 \pi K} W(t) .
\end{gathered}
$$

in the two cases, respectively. The steady-state PDF of $X$ in Equation (51) is

$$
p_{X}(x)=C \cdot \exp \left\{-\frac{1}{\pi K}\left[a \frac{x^{2}}{2}+b \frac{x^{4}}{4}\right]\right\},
$$

in which $C$ is a normalization constant.

The analyses have been accomplished with the following values of the parameters: $a=1, K=0.5$ in Equation (50); $a=-1, b=0.1, K=1 / \pi$ in Equation (51) in such a way that the PDF (52) is bimodal. In both cases the initial conditions are random, that is $p_{X}(x, 0)$ is a Gaussian PDF with zero mean and standard deviation $\sigma_{X}^{2}=0.5$. Since the two systems are scalar, only 32 finite elements suffice to get a good agreement among numerical and theoretical results, but the computations 
are repeated every $0.01 \mathrm{~s}$, that is $\Delta t=0.01$.

The time evolutions of $p_{X}(x, t)$ are in Figures 2 and 3 for Equations (50) and (51), respectively. It is not surprising that the PDF of $X$ depicted in Figure 2 converges very fast to the steady-state one as this is Gaussian like the initial one. However, the convergence is fast as in the case of the nonlinear Langevin equation even if the initial and the final PDF are quite different.
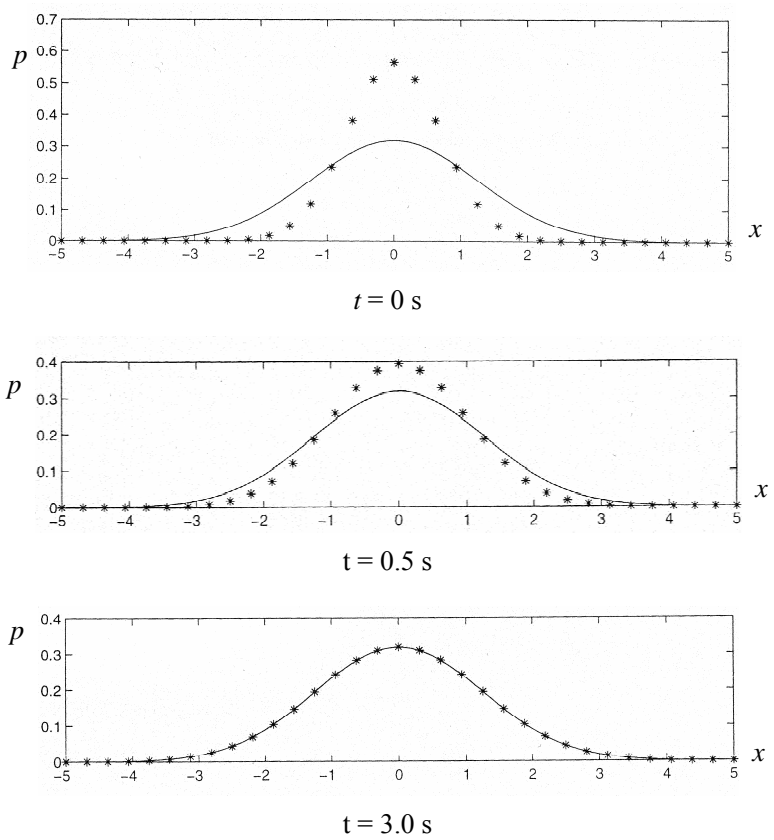

Figure 2. Time evolution of the PDF $p(x)$ of $X$ in Equation (50), random initial condition: stars FE solution, line steady-state Gaussian PDF.
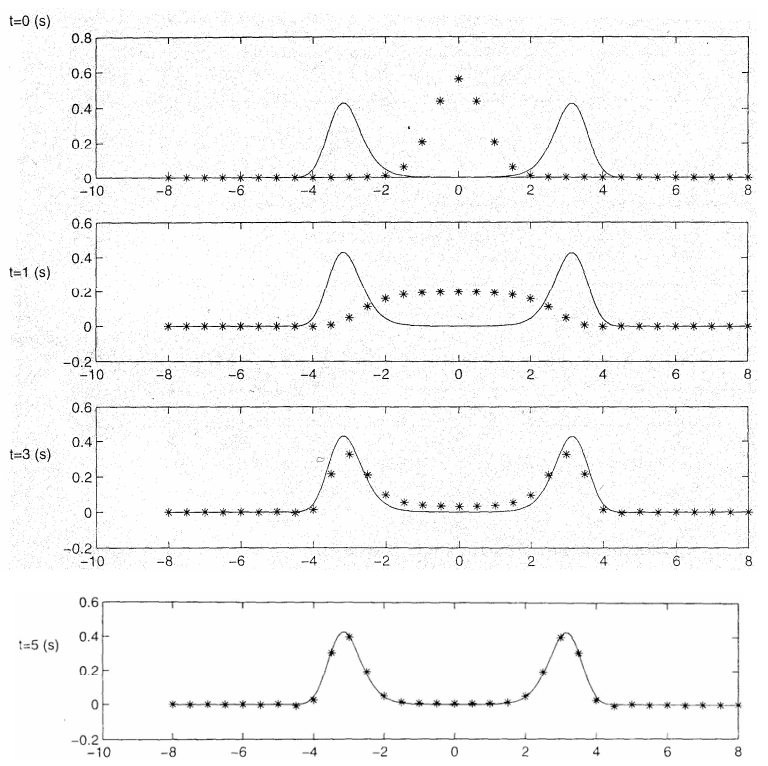

Figure 3. Time evolution of the PDF $p(x)$ of $X$ in Equation (51), random initial condition: stars FE solution, line steady-state exact PDF.

\subsection{Duffing Oscillator}

The second case that has been examined regards a Duffing oscillator excited by a Gaussian white noise. The governing equation of motion is

$$
\begin{aligned}
& \ddot{X}(t)+2 \zeta_{0} \omega_{0} \dot{X}(t)+\omega_{0}^{2}\left[\gamma X(t)+\varepsilon X^{3}(t)\right] \\
& =\sqrt{2 \pi K} W(t)
\end{aligned}
$$

where $\gamma$ and $\varepsilon$ are constant parameters, and $W(t)$ is a unit strength Gaussian white noise. In this case, the steadystate JPDF is known to be [5]

$$
p(x, \dot{x})=C \exp \left\{-\frac{\beta}{\pi K}\left[\omega_{0}^{2}\left(\gamma \frac{x^{2}}{2}+\varepsilon \frac{x^{4}}{4}\right)+\frac{\dot{x}^{2}}{2}\right]\right\},
$$

in which $\beta=2 \zeta_{0} \omega_{0}$, and $C$ is a normalization constant, whose expression is

$$
C=\left[\pi \sqrt{\frac{K}{\varepsilon \beta}} \cdot \exp \left(8 \varepsilon \sigma_{0}^{2}\right)^{-1} \cdot K_{1 / 4}\left(8 \varepsilon \sigma_{0}^{2}\right)^{-1}\right],
$$

where $\sigma_{0}^{2}=\pi K /\left(2 \zeta_{0} \omega_{0}^{3}\right)$ and $K_{1 / 4}[\bullet]$ is the modified Bessel function of order 1/4. It is recalled that, when both $\gamma$ and $\varepsilon$ are positive, the PDF of $X$ is unimodal and Gaussian like, while for $\gamma<0$ the PDF is bimodal. An analytical solution is known also for the MUR function:

$$
\begin{aligned}
v_{X}(x)= & \frac{\omega_{0} \sigma_{0} \sqrt{\varepsilon / \pi}}{K_{1 / 4}\left[\left(8 \varepsilon \sigma_{0}^{2}\right)^{-1}\right]} \cdot \exp \left(8 \varepsilon \sigma_{0}^{2}\right)^{-1} \\
& \cdot \exp \left[-\frac{1}{2 \sigma_{0}^{2}}\left(x^{2}+\varepsilon \frac{x^{4}}{4}\right)\right]
\end{aligned}
$$

The computations have been performed for $\omega_{0}=0.10 \mathrm{rad} / \mathrm{s}, \zeta_{0}=0.20, \gamma=1$ or $-1, \varepsilon=0.1, K=$ $0.4 / \pi ; 600$ elements have been used for $\gamma=-1$, while 400 or 900 for $\gamma=+1$. The statistics of $X$ are illustrated in Figures 4 and 5 for $\gamma=1$ and -1 , respectively. In both cases, the agreement is good with the exception of the MUR function of the case $\gamma=-1$, which shows a discrepancy near the peak. A visual inspection is not able to discriminate among the different approaches and degrees of approximation. Thus, the response statistical moments are to be considered.

The response statistics are listed in Tables $\mathbf{1}$ and $\mathbf{2}$ for $\gamma=1$ and $\gamma=-1$, respectively. The errors are very limited as regards the statistical moments of both $X$ and $\dot{X}$. The largest value of the MUR function $v_{X}(x)$ has a larger error, which is more pronounced in the case $\gamma=-1$. Using weighting functions different from the shape functions (WRM) yields good results; in the case $\gamma=1$ it requires a smaller number of elements, 400 against 900.

The constants $a, b$ in Equation (49) are taken equal to half the lengths of the element sides.

The values of the PDF in the tails have been con- 
trolled. As regards $p_{X}(x)$, it is worth 6.194E-04, 2.354E$07,2.577 \mathrm{E}-13$ for $x=3.0\left(\cong 3.3 \sigma_{X}\right), 4.0\left(\cong 4.4 \sigma_{X}\right)$, $5.0\left(\cong 5.5 \sigma_{X}\right)$, respectively, for $\gamma=1$. The FE method yields $1.929 \mathrm{E}-04,7.596 \mathrm{E}-08$, and $-2.635 \mathrm{E}-14$ in the three cases, respectively. Even if the errors are notable, on the other hand they are smaller than those caused by the equivalent linearization method. The estimates obtained for $\gamma=-1$ are analogous.
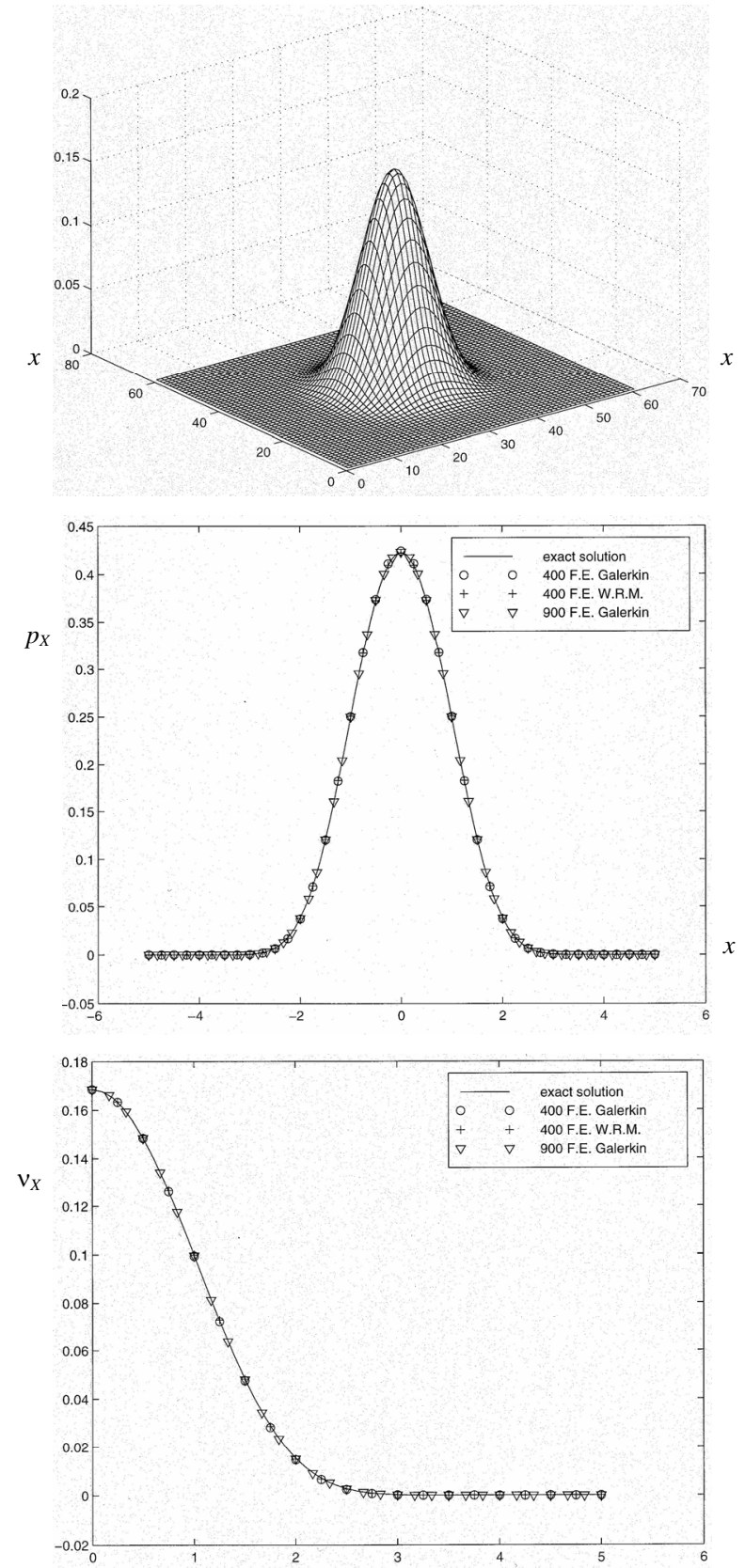

Figure 4. Steady-state statistics of $X$ for Duffing oscillator with $\gamma=1$ : top rendering of the two-dimensional JPDF of $X, \dot{X}$; middle marginal PDF of $X$; bottom MUR function $v_{X}(x)$.

\subsection{Oscillator with Parametric Excitation}

Now, consider the dynamic system

$$
\begin{aligned}
& \ddot{X}(t)+2 \zeta_{0} \omega_{0} \dot{X}(t)\left[1+\alpha X^{2}(t)\right] \\
& +\omega_{0}^{2}\left[1+W_{1}(t)\right] X(t)+F(X)=W_{2}(t)
\end{aligned}
$$

in which $W_{1}(t)$ and $W_{2}(t)$ are Gaussian uncorrelated white noises with power spectral densities $K_{1}$ and $K_{2}$, respec
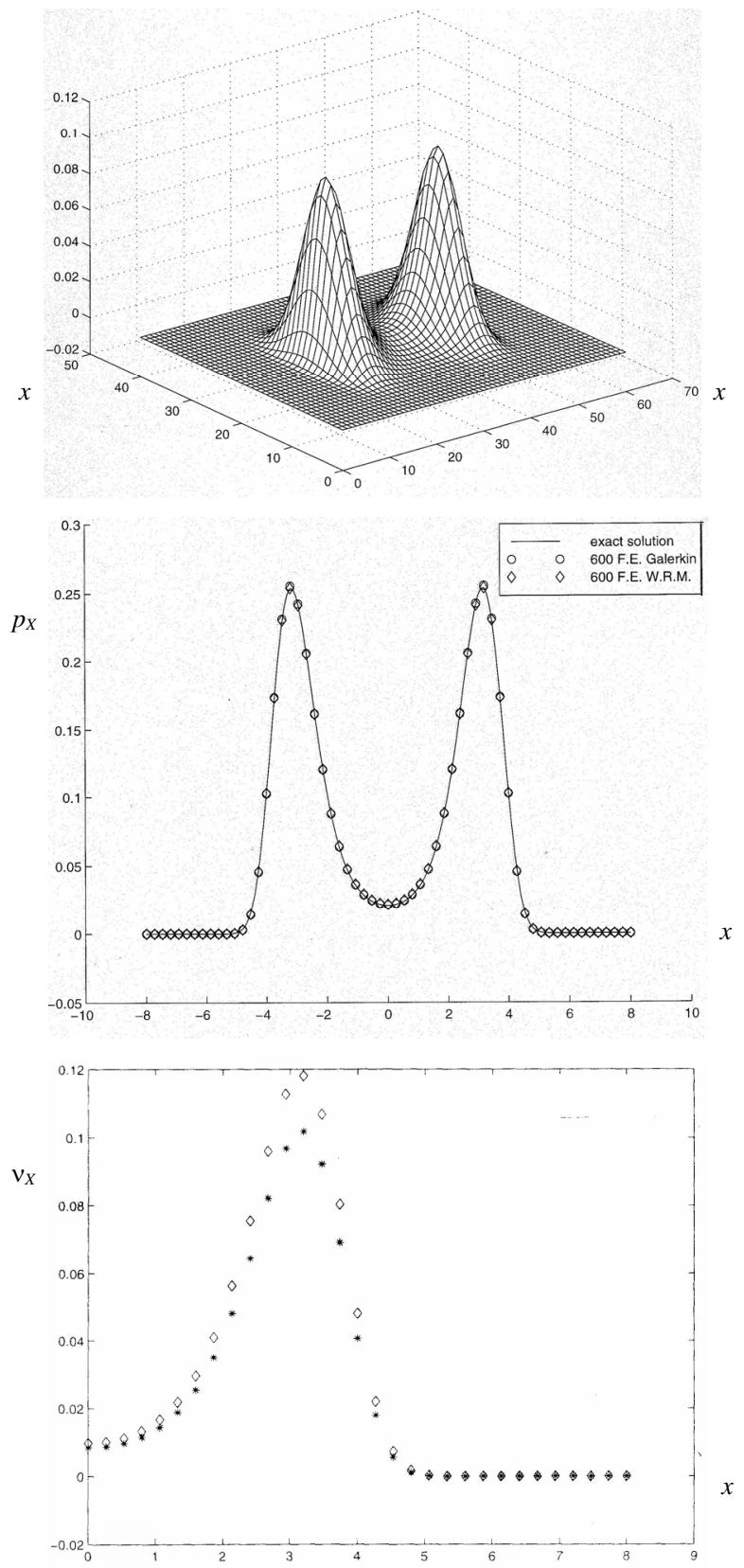

Figure 5. Steady-state statistics of $X$ for Duffing oscillator with $\gamma=-1$ : top rendering of the two-dimensional JPDF of $X, \dot{X}$; middle marginal PDF of $X$; bottom MUR function $v_{X}(x)$ (rhombs exact values, stars FE solution). 
tively. The oscillator of Equation (57), which has the parametric excitation $W_{1}(t)$, belongs to the class of generalized stationary potential [14] if the condition $\alpha=$ $\omega_{0}^{4} K_{1} / K_{2}$ is fulfilled. The steady-state JPDF has the form (27) with

$$
\phi(x, \dot{x})=\frac{2 \zeta_{0} \omega_{0}}{\pi K_{1}}\left[\frac{1}{2}\left(\dot{x}^{2}+\omega_{0}^{2} x^{2}\right)+\int_{0}^{x} f(u) \mathrm{d} u\right] .
$$

It is notable that $\phi(x, \dot{x})$ does not depend on the in-

Table 1. Response moments for $X$ of Equation (53), $\gamma=1$.

\begin{tabular}{ccccc}
\hline & $(1)$ & $(2)$ & $(3)$ & $(4)$ \\
\hline$E\left[X^{2}\right]$ & 400 & 0.806231 & 0.806231 & 0.817561 \\
& 900 & 0.812513 & 0.812513 & \\
$E\left[X^{4}\right]$ & $400^{*}$ & 0.813330 & 0.813330 & \\
& 400 & 1.760082 & 1.759952 & 1.824386 \\
$E\left[\dot{X}^{2}\right]$ & 900 & 1.795570 & 1.795544 & \\
& $400^{*}$ & 1.790557 & 1.790427 & \\
$E\left[\dot{X}^{4}\right]$ & 400 & 0.985652 & 0.985652 & 1.000 \\
& 900 & 0.996163 & 0.996163 & \\
& $400^{*}$ & 0.995807 & 0.995807 & \\
& 900 & 2.882954 & 2.882833 & 3.000 \\
$\max v_{X}(x)$ & $400^{*}$ & 3.015625 & 2.942821 & \\
& 400 & 0.168447 & 0.168433 & 0.168507 \\
& 900 & 0.168476 & 0.168473 & \\
& $400^{*}$ & 0.168517 & - &
\end{tabular}

(1) number of elements. (2) Quadratic spline. (3) Cubic spline. (4) Exact value. ${ }^{*}$ WRM.

Table 2. Response moments of $X$ in Equation (53), $\gamma=-1$.

\begin{tabular}{cccc}
\hline & $(1)$ & $(2)$ & $(3)$ \\
\hline$E\left[X^{2}\right]$ & 8.685545 & 8.68555 & 8.713629 \\
& $8.67606^{*}$ & $8.67607^{*}$ & \\
$E\left[X^{4}\right]$ & 96.3825 & 96.38228 & 97.13629 \\
& $96.3920^{*}$ & $96.39178^{*}$ & \\
$E\left[\dot{X}^{2}\right]$ & 0.996163 & 0.996163 & 1.000 \\
$E\left[\dot{X}^{4}\right]$ & 2.947204 & 2.94282 & 3.000 \\
& $3.01563^{*}$ & $3.01551^{*}$ & \\
$\max v_{x}(x)$ & 0.101775 & 0.101767 & 0.118085 \\
& $0.101702^{*}$ & - &
\end{tabular}

(1) Quadratic spline. (2) Cubic spline. (3) Exact value. "WRM. Results obtained with 600 elements. tensity of the parametric excitation $W_{1}(t)$.

The computations have been performed for the following set of parameters: $K_{1}=1\left(\mathrm{~cm}^{2} / \mathrm{s}^{3}\right)$, $K_{2}=10\left(\mathrm{~cm}^{2} / \mathrm{s}^{3}\right), \omega_{0}=2 \pi(\mathrm{rad} / \mathrm{s}), \quad \zeta_{0}=0.02$, $F(X)=\left(\omega_{0}^{2} / 10\right) \cdot \sin (2 \pi X)$. In the Bubnov-Galerkin approach, meshes with 400 and 900 elements have been tested. In the Petrov-Galerkin approach, the elements over one quarter of the integration domain are 400 , and the constants $a, b$ of Equation (49) are both 0.150 .

The exact stationary PDF of $X$ and those obtained by using the FE method are compared in the top plot of Figure 6. Again, both methods and all levels of discretization give good results so that they cannot be discriminated by a visual inspection. It is not possible to distinguish the PDF of the Bubnov-Galerkin approach from that obtained with different weighting and shape functions. The bottom plot compares the MUR functions $v_{X}(x)$. In this case, an analytical expression does not exist: the results labeled as exact are obtained inserting the exact JPDF in the Rice's formula, then the integral is numerical evaluated. The same procedure is used to obtain $v_{X}(x)$ from the FE results. The agreement is quite satisfactory.

The mean square values of $X$ and $\dot{X}$ are in Table 3 .
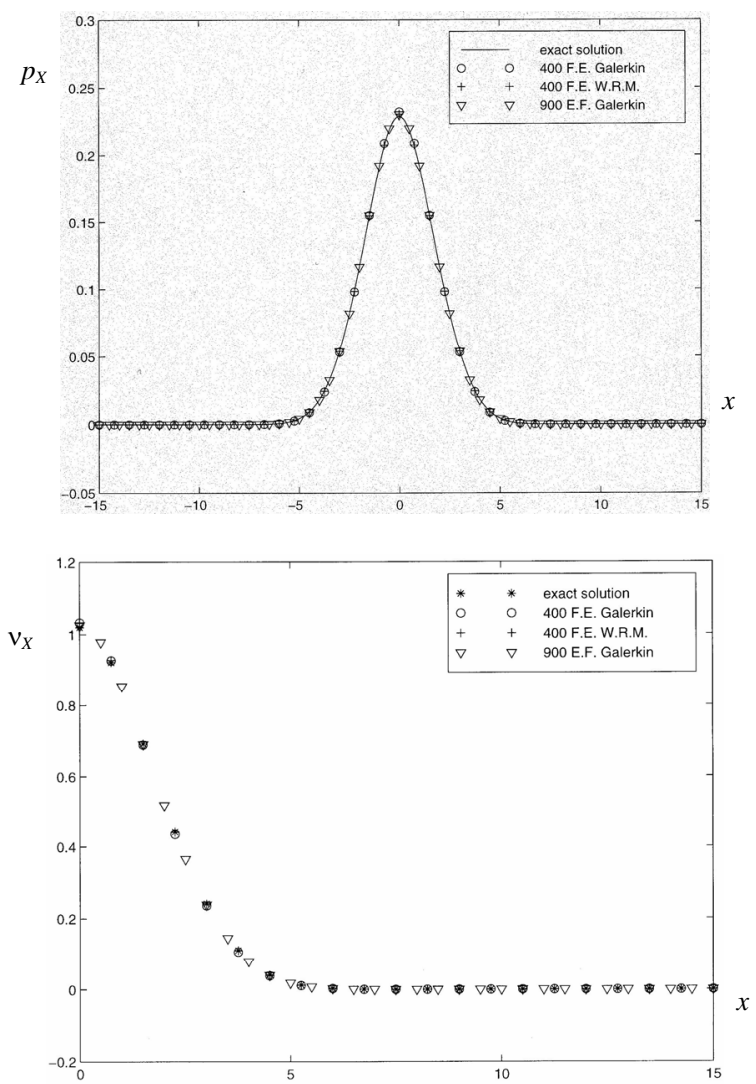

Figure 6. Steady-state statistics of $X$ in Equation (57): top marginal PDF of $X$; bottom MUR function $v_{X}(x)$ (stars exact values, circles, crosses and triangles $F E$ solutions). 
Table 3. Response moments of $X$ in Equation (57).

\begin{tabular}{cccc}
\hline & $(1)$ & $(2)$ & $(3)$ \\
\hline$E\left[X^{2}\right]$ & 3.123129 & 3.123132 & 3.252787 \\
& $3.128027^{*}$ & $3.127946^{*}$ & \\
$E\left[\dot{X}^{2}\right]$ & 124.3301 & 124.3301 & 124.9997 \\
& $125.2834^{*}$ & $125.2834^{*}$ & \\
$\max v_{X}(x)$ & 1.0244 & - & 1.0199 \\
& $1.0244^{*}$ & - & \\
\hline
\end{tabular}

(1) Simpson's rule of integration. (2) Cubic spline. (3) Exact value. ${ }^{*}$ WRM.

There are reported only the values obtained by means of 900 elements in the Bubnov's approach and 400 in the Petrov's one. It is evident that the errors are small in any case. However, Petrov's procedure allows a non negligible computing time saving.

\subsection{Oscillator with Cubic Damping and Stiffness}

Consider the following oscillator

$$
\ddot{X}(t)+\beta\left[\gamma \dot{X}(t)+\alpha \dot{X}^{3}(t)\right]+\omega_{0}^{2}\left[X(t)+\delta X^{3}(t)\right],
$$

where $\beta=2 \zeta_{0} \omega_{0}, \quad \gamma=1,-1$, and $\alpha, \delta$ are nonlinear parameters. The FPK equation associated with this oscillator does not admit an analytical solution. Thus, the finite element estimates have been compared with those obtained by means of Monte Carlo simulation. Sets with 60,000 samples of motion histories have been simulated. The parameters take the following values: $\omega_{0}=2 \pi$, $\zeta=0.02, \quad \alpha=\delta=1$.

Because of space limitations only the marginal PDF's of $X$ for $\gamma=1$ are shown in Figure 7, and only for 800 elements over one quarter of the domain with shape functions different from weighting functions. A perfect agreement between the two curves can be noted. This is confirmed by the statistical moments:

$E\left[X^{2}\right]=0.102583, \quad E\left[X^{4}\right]=0.0252177$ by simulation, and $E\left[X^{2}\right]=0.101999, E\left[X^{4}\right]=0.0249447$

by means of the FE method. However, the former requires more than 9 hours of elaboration on a workstation with quadcore processor, while the latter few minutes.

\section{Conclusions}

This paper aims at giving a contribution to some questions regarding the FE method for solving the FPK equatio $n$, which remains unanswered or partially answered. Firstly, the existence domain of a JPDF $p(z, t)$ is generally infinite, while the FE solution is restricted to a limited domain. This fact has two shortcomings: 1) the former is the necessity of having a good estimate of the response standard deviations in order to give the integration do-

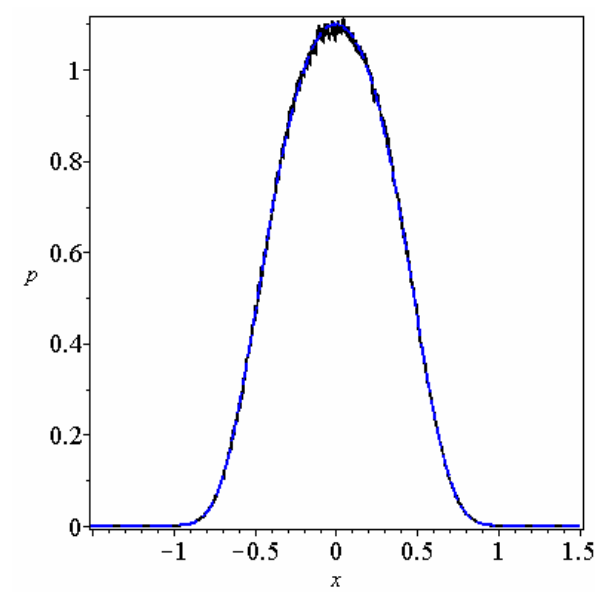

Figure 7. Steady-state PDF of $X$ in Equation (59): blue line WRM FE estimate, black line monte carlo simulation).

main appropriate dimensions; 2) no precise information is obtained on the values of the JPDF in its tails, which are very important in structural reliability. An enlargement of the domain of integration causes a notable increase of the computations in many cases. According to Langley [47], a combination of finite elements with boundary elements or infinite elements does not seem to be effective in the case of the FPK equation.

A second question is in some way related to the former: spurious oscillations of the PDF values and meaningless regions of negative values are possible, particularly in the tails, when the mesh is too coarse or badly defined. Finally, the application of the FE method to problems with more than 3 dimensions is an open question because of computer storage and speed requirements.

Computer programs have been written and implemented for both formulations of the FE method: the Bubnov-Galerkin method, which uses equal weighting and shape functions, and the Petrov-Galerkin method in which these functions are different.

Examining the results that have obtained for different stochastic systems with additive or both additive and multiplicative excitations, the following conclusions can be drawn: 1) The FE method is very feasible for solving the reduced FPK equation when the response states are few, say not more than three or four. If there are more states, programming is more cumbersome, and the computing times are unavoidably longer, not competitive with Monte Carlo simulation. 2) The steady-state statistical moments of the response are computed with small errors and a reasonable charge for computations. For two states, this is lesser than that required by a Monte Carlo simulation, and larger than that required by a moment equation approach. The FE method has the advantage that no closure schemes are required for computing the moments differently from the moment equation approach. 3) If one wants to limit the charge for computations, the 
integration domain must be kept into \pm 4 - 5 standard deviations of the variables. In this way, it is not possible to get reliable values in the tails of the JPDF, in which negative values can be encountered. 4) The use of weighting functions different from shape functions has given encouraging results so that further study in this direction seems to be promising. 5) The FE method is proved to be feasible even for transient responses, but in this case the computing time increases dramatically as the equations are to be solved in every time instant.

\section{REFERENCES}

[1] A. T. Bharucha-Reid, "Elements of Theory of Markov Processes and Their Applications," McGraw Hill, New York, 1960.

[2] R. L. Stratonovich, "Topics in the Theory of Random Noise," Gordon and Breach, New York, 1963.

[3] Y. K. Lin and G. Q. Cai, "Probabilistic Structural Dynamics: Advanced Theory and Applications," McGraw Hill, New York, 1995.

[4] H. Risken, "Fokker-Planck Equation: Methods of Solution and Applications," Springer, Berlin, 1996.

[5] T. K. Caughey, S. H. Crandall and R. H. Lyon, "Derivation and Application of the Fokker-Planck Equation to Discrete Nonlinear Dynamic Systems," Journal of Acoustical Society of America, Vol. 35, No. 11, 1963, pp. 16831692. http://dx.doi.org/10.1121/1.1918788

[6] T. K. Caughey and H. J. Payne, "On the Response of a Class of Self-Excited Oscillators to Stochastic Excitation," International Journal of Non-Linear Mechanics, Vol. 2, No. 2, 1967, pp. 125-151.

http://dx.doi.org/10.1016/0020-7462(67)90010-8

[7] A. T. Fuller, "Analysis of Nonlinear Stochastic Systems by Means of the Fokker-Planck Equation," International Journal of Control, Vol. 9, No. 6, 1969, pp. 603-655. http://dx.doi.org/10.1080/00207176908905786

[8] S. C. Liu, "Solutions of Fokker-Planck Equation with Applications in Nonlinear Random Vibration," Bell System Technical Journal, Vol. 48, No. 8, 1969, pp. 2031-2051. http://dx.doi.org/10.1002/j.1538-7305.1969.tb01163.x

[9] M. F. Dimentberg, "An Exact Solution to a Certain NonLinear Random Vibration Problem," International Journal of Non-Linear Mechanics, Vol. 17, No. 4, 1982, pp. 231-236. http://dx.doi.org/10.1016/0020-7462(82)90023-3

[10] T. K. Caughey and F. Ma, "The Steady-State Response of a Class of Dynamical Systems to Stochastic Excitation," Journal of Applied Mechanics ASME, Vol. 49, No. 3, 1982, pp. 629-632. http://dx.doi.org/10.1115/1.3162538

[11] T. K. Caughey and F. Ma, "The Exact Steady-State Solution of a Class of Non-Linear Stochastic Systems," International Journal of Non-Linear Mechanics, Vol. 17, No. 3, 1982, pp. 137-142. http://dx.doi.org/10.1016/0020-7462(82)90013-0

[12] T. K. Caughey, "On the Response of Non-Linear Oscillators to stochastic Excitation," Probabilistic Engineering
Mechanics, Vol. 1, No. 1, 1986, pp. 2-4. http://dx.doi.org/10.1016/0266-8920(86)90003-2

[13] Y. Yong and Y. K. Lin, "Exact Stationary Response Solution for Second Order Nonlinear Systems under Parametric and External White Noise Excitations," Journal of Applied Mechanics ASME, Vol. 54, No. 2, 1987, pp. 414418. http://dx.doi.org/10.1115/1.3173029

[14] Y. K. Lin and G. Q. Cai, "Exact Stationary Response Solution for Second Order Nonlinear Systems under Parametric and External White Noise Excitations: Part II," Journal of Applied Mechanics ASME, Vol. 55, No. 3, 1988, pp. 702-705. http://dx.doi.org/10.1115/1.3125852

[15] G. Q. Cai and Y. K. Lin, “On Exact Stationary Solutions of Equivalent Non-Linear Stochastic Systems," International Journal of Non-Linear Mechanics, Vol. 23, No. 4, 1988, pp. 315-325. http://dx.doi.org/10.1016/0020-7462(88)90028-5

[16] C. Soize, "Steady-State Solution of Fokker-Planck Equation in Higher Dimension," Probabilistic Engineering Mechanics, Vol. 3, No. 4, 1988, pp. 196-206. http://dx.doi.org/10.1016/0266-8920(88)90012-4

[17] W.-Q. Zhu, G. Q. Cai and Y. K. Lin, "On Exact Stationary Solutions of Stochastically Perturbed Hamiltonian Systems," Probabilistic Engineering Mechanics, Vol. 5, No. 2, 1990, pp. 84-87. http://dx.doi.org/10.1016/0266-8920(90)90011-8

[18] W.-Q. Zhu, "Exact Solutions for Stationary Responses of Several Classes of Nonlinear Systems to Parametric and/ or External White Noise Excitations," Applied Mathematics and Mechanics, Vol. 11, No. 2, 1990, pp. 165-175. http://dx.doi.org/10.1007/BF02014541

[19] C. Soize, "Exact Stationary Response of Multi-Dimensional Non-Linear Hamiltonian Dynamical Systems under Parametric and External Stochastic Excitations," Journal of Sound and Vibration, Vol. 149, No. 1, 1991, pp. 1-24. http://dx.doi.org/10.1016/0022-460X(91)90908-3

[20] W.-Q. Zhu and Y. Q. Yang, "Exact Stationary Solutions of Stochastically Excited and Dissipated Integrable Hamiltonian Systems," Journal of Applied Mechanics ASME, Vol. 63, No. 2, 1996, pp. 493-500. http://dx.doi.org/10.1115/1.2788895

[21] R. Wang and K. Yasuda, "Exact Stationary Probability Density for Second Order Nonlinear Systems under External White Noise Excitation," Journal of Sound and Vibration, Vol. 205, No. 5, 1997, pp. 647-665. http://dx.doi.org/10.1006/jsvi.1997.1052

[22] R. Wang and Z. Zhang, "Exact Stationary Response Solutions of Six Classes of Nonlinear Stochastic Systems under Stochastic Parametric and External Excitations," Journal of Engineering Mechanics ASCE, Vol. 124, No. 1, 1998, pp. 18-23. http://dx.doi.org/10.1061/(ASCE)0733-9399(1998)124:1( 18)

[23] Z. Zhang, R. Wang and K. Yasuda, "On Joint Stationary Probability Density Function of Nonlinear Dynamic Systems," Acta Mechanica, Vol. 130, No. 1, 1998, pp. 29-39. http://dx.doi.org/10.1007/BF01187041

[24] R. Wang, K. Yasuda and Z. Zhang, “A Generalized Analysis Technique of the Stationary FPK Equation in Non- 
linear Systems under Gaussian White Noise Excitations," International Journal of Engineering Science, Vol. 38, No. 12, 2000, pp. 1315-1330. http://dx.doi.org/10.1016/S0020-7225(99)00081-6

[25] R. Wang and Z. Zhang, "Exact Stationary Solutions of the Fokker-Planck Equation for Nonlinear Oscillators under Stochastic Parametric and External Exctations," Nonlinearity, Vol. 13, No. 3, 2000, pp. 907-920.

[26] Z. L. Huang and W.-Q. Zhu, "Exact Stationary Solutions of Stochastically and Harmonically Excited and Dissipated Integrable Hamiltonian Systems," Journal of Sound and Vibration, Vol. 230, No. 3, 2000, pp. 709-720. http://dx.doi.org/10.1006/jsvi.1999.2634

[27] W.-Q. Zhu and Z. L. Huang, "Exact Stationary Solutions of Stochastically Excited and Dissipated Integrable Hamiltonian Systems," International Journal of Non-Linear Mechanics, Vol. 36, No. 1, 2001, pp. 3-48. http://dx.doi.org/10.1016/S0020-7462(99)00086-4

[28] R. G. Bhandari and R. E. Sherrer, "Random Vibration in Discrete Nonlinear Dynamic Systems," Journal of Mechanical Engineering Science, Vol. 10, No. 2, 1968, pp. 168-174.

http://dx.doi.org/10.1243/JMES JOUR $1968 \quad 010 \quad 024 \quad 0$ $\underline{2}$

[29] G. Muscolino, G. Ricciardi and M. Vasta, "Stationary and Non-Stationary Probability Density Function for NonLinear Oscillators," International Journal of Non-Linear Mechanics, Vol. 32, No. 6, 1997, pp. 1051-1064. http://dx.doi.org/10.1016/S0020-7462(96)00134-5

[30] G.-K. Er, "Multi-Gaussian Closure Method for Randomly Excited Non-Linear Systems," International Journal of Non-Linear Mechanics, Vol. 33, No. 2, 1998, pp. 201-214. http://dx.doi.org/10.1016/S0020-7462(97)00018-8

[31] G.-K. Er, "A Consistent Method for the Solution to Reduced FPK Equation in Statistical Mechanics," Physica A, Vol. 262, No. 1-2, 1999, pp. 118-128. http://dx.doi.org/10.1016/S0378-4371(98)00362-8

[32] M. Di Paola and A. Sofi, "Approximate Solution of the Fokker-Planck-Kolmogorov Equation," Probabilistic Engineering Mechanics, Vol. 17, No. 4, 2002, pp. 369-384. http://dx.doi.org/10.1016/S0266-8920(02)00034-6

[33] W. Martens, U. von Wagner and V. Mehrmann, "Calculation of High-Dimensional Probability Density Functions of Stochastically Excited Nonlinear Mechanical Systems," Nonlinear Dynamics, Vol. 67, No. 3, 2012, pp. 2089-2099. http://dx.doi.org/10.1007/s11071-011-0131-2

[34] J. D. Atkinson, "Eigenfunction Expansions for Randomly Excited Non-Linear Systems," Journal of Sound and Vibration, Vol. 30, No. 2, 1973, pp. 153-172. http://dx.doi.org/10.1016/S0022-460X(73)80110-5

[35] Y.-K. Wen, "Approximate Method for Nonlinear Random Vibration," Journal of Engineering Mechanics Division ASCE, Vol. 101, No. 4, 1975, pp. 389-401.

[36] J. P. Johnson and R. A. Scott, "Extension of Eigenfunction Expansion of a Fokker-Planck Equation-I. First Order System," International Journal of Non-Linear Mechanics, Vol. 14, No. 5-6, 1979, pp. 315-324. http://dx.doi.org/10.1016/0020-7462(79)90005-2
[37] J. P. Johnson and R. A. Scott, "Extension of Eigenfunction Expansion of a Fokker-Planck Equation-II. Second Order System," International Journal of Non-Linear Mechanics, Vol. 15, No. 1, 1980, pp. 41-56. http://dx.doi.org/10.1016/0020-7462(80)90052-9

[38] U. von Wagner and W. V. Wedig, "On the Calculation of Stationary Solutions of Multi-Dimensional Fokker-Planck Equations by Orthogonal Functions," Nonlinear Dynamics, Vol. 21, No. 3, 2000, pp. 289-306. http://dx.doi.org/10.1023/A:1008389909132

[39] R. Courant and D. Hilbert, "Methods of Mathematical Physics," John Wiley \& Sons, New York, 1989.

[40] J. B. Roberts, "First-Passage Time for Randomly Excited Non-Linear Oscillators," Journal of Sound and Vibration, Vol. 109, No. 1, 1986, pp. 33-50. http://dx.doi.org/10.1016/S0022-460X(86)80020-7

[41] T. Blum and A. J. McKane, "Variational Schemes in the Fokker-Planck Equation," Journal of Physics A: Mathematical and General, Vol. 29, No. 9, 1996, pp. 18591872. http://dx.doi.org/10.1088/0305-4470/29/9/003

[42] M. Dehghan and M. Tatari, "The Use of He's Variational Iteration Method for Solving a Fokker-Planck Equation", Physica Scripta, Vol. 74, No. 3, 2006, pp. 310-316. http://dx.doi.org/10.1088/0031-8949/74/3/003

[43] A. Quarteroni and A. Valli, "Numerical Approximation of Partial Differential Equations," Springer, Berlin, 1994.

[44] L. A. Bergman and J. C. Heinrich, "Solution of the Pontriagin-Vitt Equation for the Moments of Time to First Passage of the Randomly Accelerated Particle by the Finite Element Method," International Journal for $\mathrm{Nu}$ merical Methods in Engineering, Vol. 15, No. 9, 1980, pp. 1408-1412. http://dx.doi.org/10.1002/nme.1620150913

[45] L. A. Bergman and J. C. Heinrich, "On the Moments of Time to First Passage of Linear Oscillator," Earthquake Engineering and Structural Dynamics, Vol. 9, No. 3, 1981, pp. 197-204. http://dx.doi.org/10.1002/eqe.4290090302

[46] B. F. Spencer Jr. and L. A. Bergman, "On the Reliability of a Simple Hysteretic System," Journal of Engineering Mechanics, Vol. 111, No. 12, 1985, pp. 1502-1514. http://dx.doi.org/10.1061/(ASCE)0733-9399(1985)111:12 (1502)

[47] R. S. Langley, "A Finite Element Method for the Statistics of Non-Linear Random Vibration," Journal of Sound and Vibration, Vol. 101, No. 1, 1985, pp. 41-54. http://dx.doi.org/10.1016/S0022-460X(85)80037-7

[48] H. P. Langtangen, "A General Numerical Solution Method for Fokker-Planck Equations with Applications to Structural Reliability," Probabilistic Engineering Mechanics, Vol. 6, No. 1, 1991, pp. 33-48. http://dx.doi.org/10.1016/S0266-8920(05)80005-0

[49] B. F. Spencer Jr. and L. A. Bergman, "On the Numerical Solution of the Fokker-Planck Equation for Nonlinear Stochastic Systems," Nonlinear Dynamics, Vol. 4, No. 4, 1993, pp. 357-372.

http://dx.doi.org/10.1007/BF00120671

[50] H. U. Köylüoglu, S. R. K. Nielsen and R. Iwankiewicz, "Reliability of Non-Linear Oscillators Subject to Poisson 
Driven Impulses," Journal of Sound and Vibration, Vol. 176, No. 1, 1994, pp. 19-33. http://dx.doi.org/10.1006/jsvi.1994.1356

[51] L.-C. Shiau and T.-Y. Wu, "A Finite Element Method for Analysis of Non-Linear System under Stochastic ParaMetric and External Excitation," International Journal of Non-Linear Mechanics, Vol. 31, No. 2, 1996, pp. 193201. http://dx.doi.org/10.1016/0020-7462(95)00049-6

[52] L. A. Bergman, S. F. Wojtkiewicz, E. A. Johnson and B. F. Spencer Jr., "Robust Numerical Solution of the Fokker-Planck Equation for Second Order Dynamical Systems under Parametric and External White Noise Excitation," In: W. H. Kliemann, Ed., Nonlinear Dynamics and Stochastic Mechanics, American Mathematical Society, Providence, 1996, pp. 23-37.

[53] A. Masud and L. A. Bergman, "Application of Multi-Scale Finite Element Methods to the Solution of the FokkerPlanck Equation," Computer Methods in Applied Mechanics and Engineering, Vol. 194, No. 12-16, 2005, pp. 15131526. http://dx.doi.org/10.1016/j.cma.2004.06.041

[54] M. Kumar, S. Chakravorty, P. Singla and J. L. Junkins, "The partition of Unity Finite Element Approach with Hp-Refinement for the Stationary Fokker-Planck Equation," Journal of Sound and Vibration, Vol. 327, No. 1-2,
2009 , pp. 144-162.

http://dx.doi.org/10.1016/j.jsv.2009.05.033

[55] E. Wong and M. Zakai, "On the Relation between Ordinary and Stochastic Equations," International Journal of Engineering Science, Vol. 3, No. 2, 1965, pp. 213-229. http://dx.doi.org/10.1016/0020-7225(65)90045-5

[56] R. L. Stratonovich, "Topics in Theory of Random Noise," Taylor \& Francis, New York, 1967.

[57] K. Itô, "On Stochastic Differential Equations," Memoirs of American Mathematical Society, Vol. 4, 1951, pp. 1-51.

[58] K. Itô, "On a Formula Concerning Stochastic Differentials," Nagoya Mathematical Journal, Vol. 3, No. 1, 1951, pp. 55-65.

[59] M. Di Paola, "Stochastic Differential Calculus," In: F. Casciati, Ed., Dynamic Motion: Chaotic and Stochastic Behaviour, Springer Verlag, Wien, 1993, pp. 29-92.

[60] W. Q. Zhu, "Nonlinear Stochastic Dynamics and Control in Hamiltonian Formulation," Applied Mechanics Review, Vol. 59, No. 4, 2006, pp. 230-248. http://dx.doi.org/10.1115/1.2193137

[61] J. B. Roberts and P. Spanos, "Random Vibration and Statistical Linearization,” John Wiley \& Sons, New York, 1990. 